\title{
Regional Differences in Noradrenaline-Induced Release of Adenosine Triphosphate from Rat Vascular Endothelium
}

\author{
Kazumasa Shinozuka, Michio Hashimoto*, Young Mi Kwon, \\ Sumio Masumura*, Richard A. BJUR**, David P. Westfall** \\ and Masaru Kunitomo \\ Department of Pharmacology, Faculty of Pharmaceutical Sciences, Mukogawa \\ Women's University, Nishinomiya, 663-8179 Japan. \\ *Department of Physiology, Shimane Medical University, Izumo, 693-8501 \\ Japan. \\ **Department of Pharmacology, University of Nevada School of Medicine, \\ Reno, NV 89557-0046, USA.
}

\begin{abstract}
We examined the release of endogenous adenyl purines such as adenosine triphosphate (ATP), ADP, AMP and adenosine from the caudal artery (CA), saphenous artery (SA), renal artery (RA), mesenteric artery (MA), pulmonary artery (PA) and thoracic aorta (TA) of rats, using high-performance liquid chromatography fluorescence detection. Noradrenaline induced the release of adenyl purines from these blood vessels. The total amount of adenyl purines release induced by noradrenaline from the CA was considerably larger than that from the TA. The rank order of the amount of adenyl purines released from the six blood vessels was $\mathrm{CA}>\mathrm{SA}>\mathrm{RA}>\mathrm{MA}>\mathrm{PA} \geqq \mathrm{TA}$. The noradrenaline-induced release of adenyl purines from the $\mathrm{CA}$ was significantly reduced by the removal of the endothelium. Noradrenaline also induced the release of adenyl purines from cultured endothelial cells of the $\mathrm{CA}$ and TA. The total amount of adenyl purines released from the former blood vessel was much larger than that from the latter. These results suggest the existence of vascular endothelial cells that are able to release ATP by an $\alpha_{1}$-adrenoceptor mediated mechanism, and that these cells are not homogeneously distributed in the vasculature.
\end{abstract}

Key Words: ATP-rat blood vessels-endothelial cells- $\alpha_{1}$-adrenoceptor-regional difference

\section{Introduction}

Adenosine triphosphate (ATP), in addition to its known functions in intracellular metabolism, is increasingly being recognized for its extracellular functions in various tissues, including blood vessels (Gordon, 1986; White, 1988 ; Burnstock, 1991). There have been many reports that adenine nucleotides and nucleosides are released from a variety of sympathetically inner-

Correspondence to: Kazumasa Shinozuka, Department of Pharmacology, Faculty of Pharmaceutical Sciences, Mukogawa Women's University, Nishinomiya, Hyogo Pref. 663-8179, Japan.

Phone : 0798-47-1212 
vated tissues upon nerve stimulation. It is generally accepted that ATP was released from adrenergic nerves with noradrenaline as a co-transmitter in electrically stimulated tissues such as the vas deferens (Sneddon and Westfall, 1984) and blood vessels (Su, 1975, 1983 ; Sneddon and Burnstock, 1985). We have shown that the stimulation of the $\alpha_{1}$-adrenoceptor induced the release of ATP from the endothelium of the rat caudal artery (Westfall et al., 1987 ; Shinozuka et al., 1991, 1994). In view of the fact that ATP, ADP and adenosine have many pharmacological actions on blood vessels via purinoceptors, the endothelium-derived ATP and its metabolites may play important physiological roles in the cardiovascular system. However, there are very few reports regarding this. As an initial approach to clarify the physiological significance of ATP and its metabolites released from endothelium upon $\alpha_{1}$-adrenoceptor stimulation, we examined the regional differences of adenyl purine release in several blood vessels isolated from the rat, using high-performance liquid chromatography (HPLC)-fluorescence detection.

\section{Methods}

Tissue preparations and release experiment

Male Wistar rats (SLC, Hamamatsu, Japan) ranging from 20 to 25 weeks of age were used in accordance with the Guidelines for Animal Experimentation of Shimane Medical University, which are based on the Guidelines for Animal Experimentation of the Japanese Association for Laboratory Animal Science. The caudal artery (CA), saphenous artery (SA), renal artery (RA), mesenteric artery (MA), pulmonary artery (PA) and thoracic aorta (TA) were isolated from the rats, which had been anesthetized with sodium pentobarbital $(50 \mathrm{mg} / \mathrm{kg}$ intraperitoneally). These blood vessel preparations were cut open lengthwise and suspended in a water-jacketed organ chamber containing $3.0 \mathrm{ml}$ of a modified Krebs solution at $37^{\circ} \mathrm{C}$. The solution was continuously bubbled with $95 \% \mathrm{O}_{2}$ and $5 \% \mathrm{CO}_{2}$. The composition of the solution was as follows: $(\mathrm{mM}) ; \mathrm{NaCl} 110 ; \mathrm{KCl} 4.6 ; \mathrm{CaCl}_{2} 2.5 ; \mathrm{NaHCO}_{3} 24.8 ; \mathrm{KH}_{2} \mathrm{PO}_{4} 1.2 ; \mathrm{MgSO}_{4}$ 1.2 ; glucose 5.6. The opened artery preparation was then allowed to equilibrate for $60 \mathrm{~min}$, and the medium was replaced every 3 min during the latter half of this equilibration period. Some experiments were carried out with the opened caudal artery preparation in which the endothelial cell lining was disrupted. This was accomplished by rubbing the lumen of the arterial segment with a cotton-tipped swab.

After the 60-min equilibration period, the bathing solution was rapidly collected by draining the organ chamber every $3 \mathrm{~min}$. After the first sampling (prestimulation sample), the tissues were stimulated with noradrenaline $(1.0 \mu \mathrm{M})$ for $3 \mathrm{~min}$, and then the bathing solutions were collected (stimulation samples). The collected samples were then processed for the determination of ATP, ADP, AMP and adenosine by HPLC coupled with fluorescence detection as described by Mohri et al. (1993).

\section{Cell cultures and release experiment}

Endothelial cells in primary cultures from either the thoracic aorta or caudal artery of the rat were prepared by the methods previously described (Hashimoto et al., 1992). The cells in 
the primary cultures were incubated in $2 \mathrm{ml}$ of Krebs solution for $60 \mathrm{~min}$ at $37^{\circ} \mathrm{C}$ in an atmosphere of $95 \%$ air and $5 \% \mathrm{CO}_{2}$. After $60 \mathrm{~min}$ of equilibration, $200 \mu \mathrm{l}$ of bathing solution was collected (prestimulation sample) and the endothelial cells were then stimulated with noradrenaline for $3 \mathrm{~min}$, followed by another $200 \mu \mathrm{l}$ collection (stimulation sample). The collected samples were then processed for the determination of adenine nucleotides and adenosine as described previously (Mohri et al., 1993).

\section{Statistical analysis}

All data are expressed as the means \pm S.E.M (standard error of the mean). Data were evaluated for significance by one-way analysis of variance (ANOVA) combined with Fisher's PLSD test for multiple comparison or Student's t-test, and the Welch test where appropriate. Values with a $\mathrm{P}<0.05$ were considered to be significantly different. Data processing was performed with the computer software package "Stat View ${ }^{\mathrm{TM}}$ II" (Abacus Concepts, Inc., Berkeley, CA, USA).

\section{Results}

The spontaneous overflows of ATP, ADP, AMP and adenosine from the caudal artery (CA), saphenous artery (SA), renal artery (RA), mesenteric artery (MA), pulmonary artery (PA)

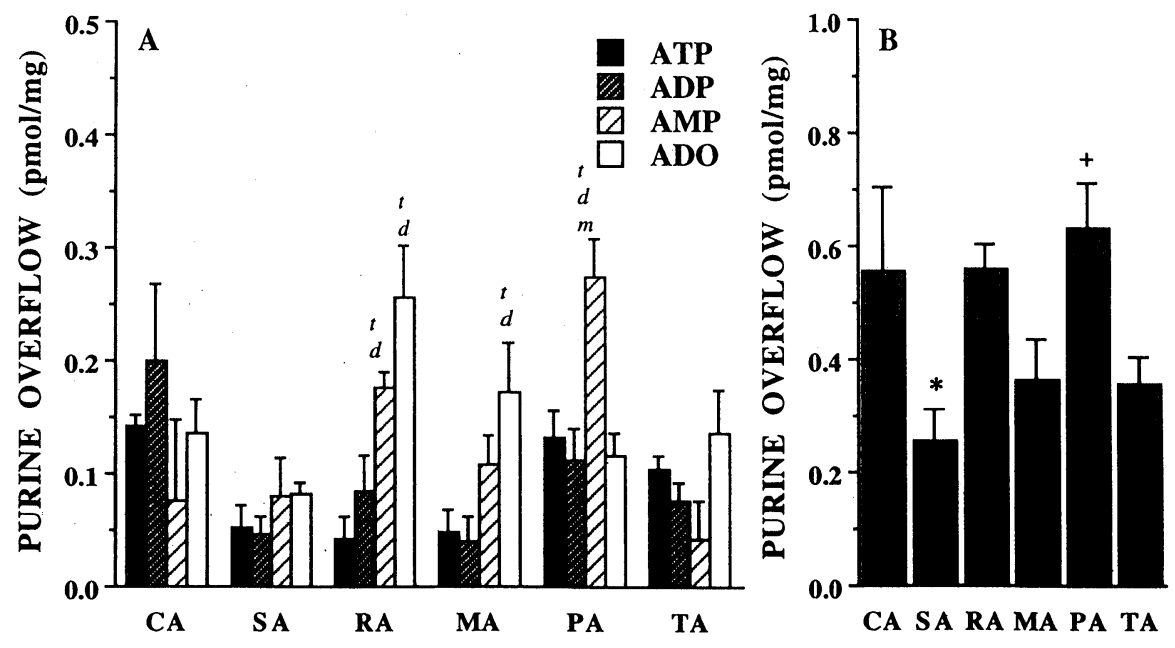

Fig. 1. Comparison of the spontaneous overflow of adenyl purines from the caudal artery (CA), saphenous artery (SA), renal artery (RA), mesenteric artery (MA), pulmonary artery (PA) and thoracic aorta (TA). The vertical bars on the columns indicate the S.E.M. $(n=4)$. Left panel $(\mathrm{A})$ : the spontaneous overflow amounts of each purine from the artery or aorta during a 3 -min period. $t, d$ and $m$ indicate significant differences $(\mathrm{P}<0.05)$ from ATP, ADP and AMP, respectively, in each blood vessel. Right panel (B) : the amounts of total purine refers to the sum total overflow of the four purines (ATP, ADP, AMP and adenosine $(\mathrm{ADO}))$. *Significant difference $(\mathrm{P}<0.05)$ from $\mathrm{CA}, \mathrm{RA}$ and $\mathrm{PA} .{ }^{+}$Significant difference $(\mathrm{P}<0.05)$ from MA and TA. 
and thoracic aorta (TA) are shown in Fig. 1. There were no significant differences among the four purines, such as ATP, ADP, AMP and adenosine, in CA, SA and TA (Fig. 1A). In RA and MA, the overflow amounts of ATP and ADP were significantly smaller than those of AMP and adenosine. Further, in PA, the overflow amount of AMP was largest. As shown in Fig. 1B, the total amount of adenyl purine overflow from the SA was significantly smaller than those from the CA, RA and MA. In addition, the total amounts of purine overflow from the MA and TA were significantly smaller than that from the PA. There was no significant difference among the amounts of the overflow from the CA, RA and PA, or among those from the SA, MA and TA. In all six blood vessels, CA, SA, RA, MA, PA and TA, noradrenaline $(1 \mu \mathrm{M})$ significantly increased the total overflow of adenine nucleotides and nucleoside from $0.56 \pm 0.15$, $0.26 \pm 0.06,0.56 \pm 0.05,0.37 \pm 0.07,0.63 \pm 0.08$ and $0.36 \pm 0.05$ to $8.59 \pm 0.78,6.25 \pm 0.53,3.58 \pm 0.40$, $1.85 \pm 0.07,1.00 \pm 0.13$ and $0.46 \pm 0.05 \mathrm{pmol} / \mathrm{mg}$, respectively $(\mathrm{P}<0.05$, paired $\mathrm{t}$-test; $\mathrm{n}=4)$. Figure 2 shows the release of adenyl purines from the vessels induced by noradrenaline. The release indicates the difference between the amounts of overflow of adenyl purines in the prestimulation and stimulation samples (noradrenaline-induced overflow minus spontaneous overflow). As shown in Fig. 2A, the amounts of ATP and ADP released were significantly smaller than those of AMP and adenosine in CA, SA, RA and MA. In PA, the amount of AMP released was the largest. The total amount of purine release from the CA was significantly larger than those from the other five blood vessels (Fig. 2B). The rank order of the nora-
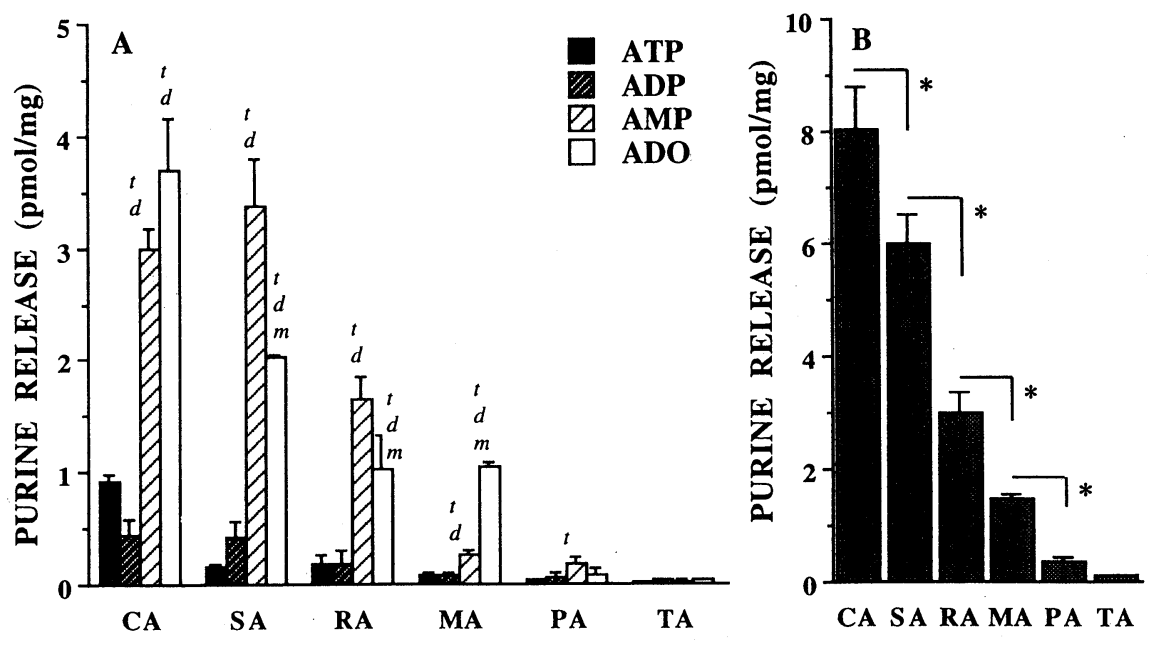

Fig. 2. Comparison of the noradrenaline-induced release of adenyl purines from the caudal artery (CA), saphenous artery (SA), renal artery (RA), mesenteric artery (MA), pulmonary artery $(\mathrm{PA})$ and thoracic aorta (TA). The release indicates the difference between the amount of adenyl purines in prestimulation (in the absence of noradrenaline) and post stimulation (in the presence of noradrenaline) samples. The vertical bars on the columns indicate the S.E.M. $(\mathrm{n}=4)$. Left panel $(\mathrm{A})$ : the amounts of each purine released from the artery or aorta during a 3 -min period. $t, d$ and $m$ indicate significant differences $(\mathrm{P}<0.05)$ from ATP, ADP and AMP, respectively, in each blood vessel. Right panel (B) : the amounts of total purine which refers to the sum total of the four purines release (ATP, ADP, AMP and adenosine $(\mathrm{ADO}))$. ${ }^{*}$ Significant difference $(\mathrm{P}<0.05)$. 
drenaline-induced amount of total adenyl purine release from the six blood vessels was $\mathrm{CA}>$ $\mathrm{SA}>\mathrm{RA}>\mathrm{MA}>\mathrm{PA} \geqq \mathrm{TA}$. There was no significant difference between the amounts of the release from the PA and TA. In the TA, the amount of release (pmol/mg) was only approximately $1 / 80$ of that obtained from the CA. When the amount of release was compared on the basis of area $\left(\mathrm{pmol} / \mathrm{mm}^{2}\right)$ instead of weight of tissue $(\mathrm{pmol} / \mathrm{mg})$, the amounts of purine released by the CA, SA and RA $\left(0.96 \pm 0.08,0.79 \pm 0.06\right.$ and $0.44 \pm 0.05 \mathrm{pmol} / \mathrm{mm}^{2}, \mathrm{n}=4$, respectively) were still significantly larger than those released by the MA, PA and TA $(0.17 \pm 0.01,0.04 \pm 0.01$ and $0.03 \pm 0.01 \mathrm{pmol} / \mathrm{mm}^{2}, \mathrm{n}=4$, respectively). In the $\mathrm{CA}$, adenosine was present in the largest quantity, and was approximately equal in amount to the sum of the nucleotides ATP, ADP and AMP (Fig. 2A).

Figure 3 and 4 show the spontaneous overflow and the noradrenaline-induced release of adenine nucleotides and nucleoside from the $\mathrm{CA}$ in which the endothelium was left intact or was removed by rubbing the lumen, respectively. There was no significant difference between the total amounts of adenyl purine spontaneous overflow from the intact and rubbed CA (Fig. 3). However, the total amount of purine release induced by noradrenaline from the rubbed CA was significantly smaller than that from the intact CA (Fig. 4). The former was approximately $30 \%$ of the latter.

The spontaneous overflow of adenyl purines from the cultured endothelial cells which had been isolated from the CA or TA is shown in Fig. 5. There was no significant difference between the spontaneous overflow levels of adenyl purines from these cultured endothelial cells. In the cells $\mathrm{TA}$ and $\mathrm{CA}$, noradrenaline $(10.0 \mu \mathrm{M})$ significantly increased the overflow of total purines from $1.38 \pm 0.46$ and $1.46 \pm 0.46$ to $1.58 \pm 0.46$ and $4.92 \pm 1.45 \mathrm{pmol} / \mathrm{mg}$, respectively $(\mathrm{P}<$ 0.05 , paired $\mathrm{t}$-test; $\mathrm{n}=4$ ). However, the noradrenaline-induced release of purine by the cultured endothelial cells from the CA was again much larger than that from the TA, with the
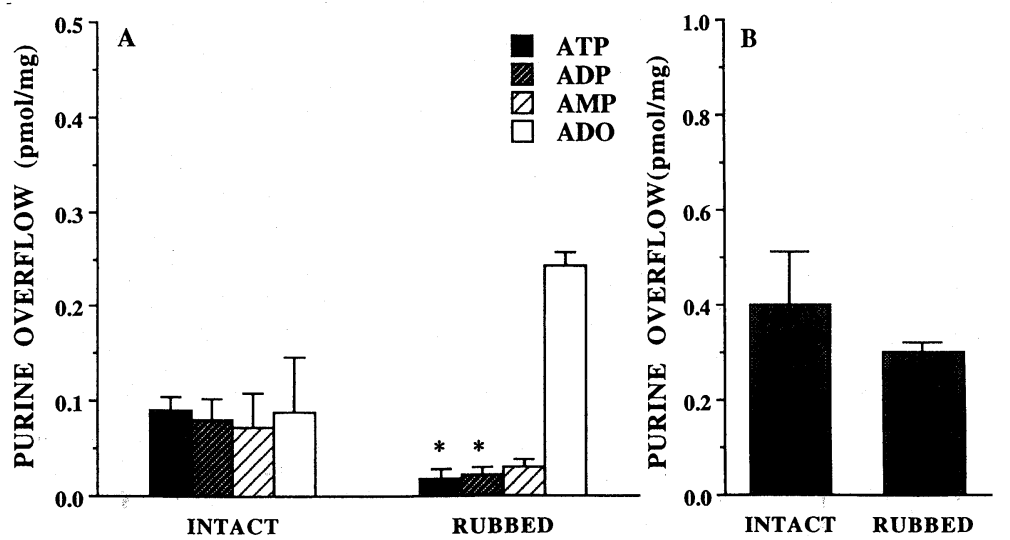

Fig. 3. Comparison of the spontaneous overflow of adenyl purines from intact and rubbed caudal artery of rats. The vertical bars on the columns indicate the S.E.M. $(n=4)$. *Significant difference $(\mathrm{P}<0.05)$ from each purine in intact. Left panel $(\mathrm{A})$ : the amounts of each purine released from the artery during a 3-min period. Right panel (B) : the amounts of total purine refers to the sum total of the four purines released (ATP, ADP, AMP and adenosine $(\mathrm{ADO}))$. * *Significant difference $(\mathrm{P}<0.05)$. 

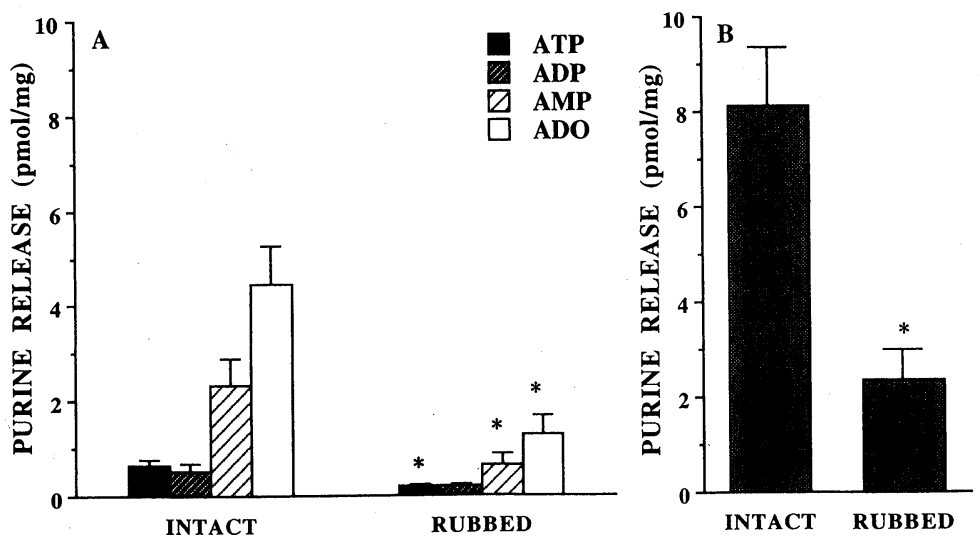

Fig. 4. Comparison of the noradrenaline-induced release of adenyl purines from intact and rubbed caudal artery of rats. The vertical bars on the columns indicate the S.E.M. $(n=4)$. *Significant difference $(\mathrm{P}<0.05)$ from each purine level in intact vessels. Left panel $(\mathrm{A})$ : the amounts of each purine released from the artery during a 3-min period. Right panel : the amounts of total purine refers to the sum total of the four purines released (ATP (B), ADP, AMP and adenosine $(\mathrm{ADO}))$. * ${ }^{*}$ Significant difference $(\mathrm{P}<0.05)$.
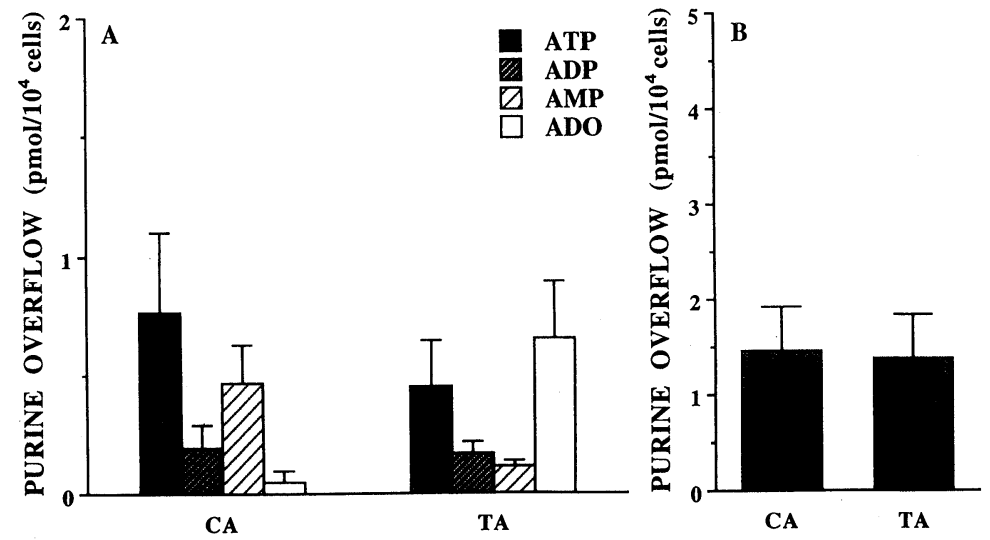

Fig. 5. Comparison of the spontaneous overflow of adenyl purines from the endothelial cells in primary culture. Cells were prepared from the caudal artery (CA) and thoracic aorta (TA) of rats. The vertical bars on the columns indicate the S.E.M. $(n=4)$. Left panel $(A)$ : the overflow amounts of each purine from the artery or aorta during a 3-min period. Right panel $(B)$ : the amounts of total purine refers to the sum total overflow of the four purines (ATP, ADP, AMP and adenosine (ADO)).

former approximately 10 times the latter (Fig. 6). This release of purines from specific endothelial cell populations is therefore in agreement with the results obtained with intact CA and TA. As shown in Fig. $6 \mathrm{~A}$, the amount of ATP $\left(1.95 \pm 0.68 \mathrm{pmol} / 10^{4}\right.$ cells $)$ released from cultured endothelial cells from the $\mathrm{CA}$ by noradrenaline was significantly $(\mathrm{P}<0.05)$ larger than that of adenosine $\left(0.03 \pm 0.02 \mathrm{pmol} / 10^{4}\right.$ cells $)$. 

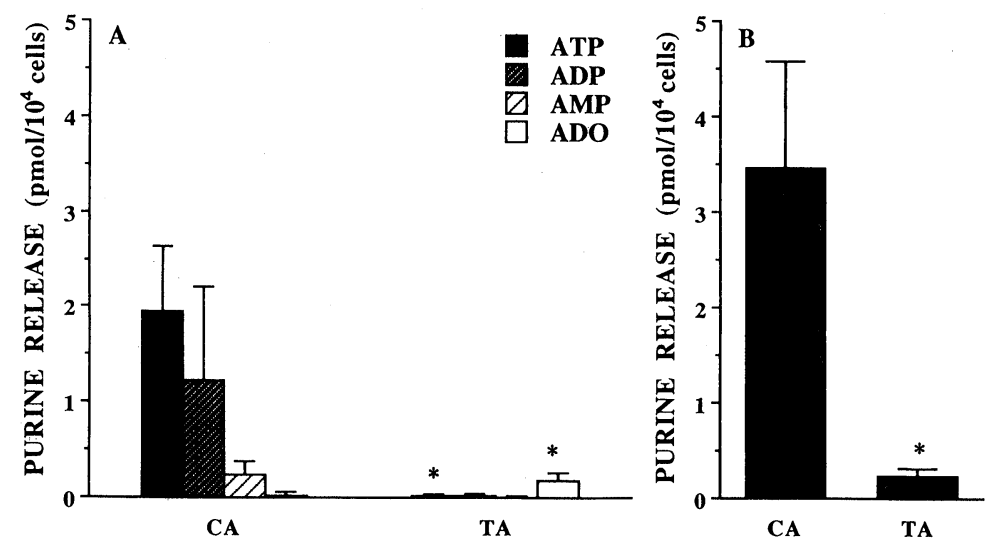

Fig. 6. Comparison of spontaneous overflow and noradrenaline-induced release of adenyl purines from endothelial cells in primary culture. Cells were prepared from the caudal artery (CA) and thoracic aorta (TA) of rats. The vertical bars on the columns indicate the S.E. M. $(\mathrm{n}=4)$. * Significant difference $(\mathrm{P}<0.05)$ from each purine level in CA. Left panel $(\mathrm{A})$ : the amounts of each purine released from the artery or aorta during a 3-min period. Right panel (B) : the amounts of total purine refers to the sum total of the four purines released (ATP, ADP, AMP and adenosine (ADO)).

\section{Discussion}

Previous study at our laboratories (Shinozuka et al., 1994) has indicated that noradrenaline released ATP and its metabolites via the stimulation of $\alpha_{1}$-adrenoceptors in the rat caudal artery. The present experimental results also suggest that a functional role exists for an $\alpha_{1}-$ adrenoceptor-mediated release of adenyl purines that is regionally differentiated in the vasculature. The rank order of the amounts of total adenyl purines released by noradrenaline from the six blood vessels was caudal artery $>$ saphenous artery $>$ renal artery $>$ mesenteric artery $>$ renal artery $\geqq$ thoracic aorta. These release values were compared on the bases of both $\mathrm{pmol} / \mathrm{mg}$ wet weight and $\mathrm{pmol} / \mathrm{mm}^{2}$ area of tissue analyzed. The differences among the amount of purines released from the six blood vessels thus do not appear to be due to differences in the area of the intima layer (i.e., the number of endothelial cells).

The present results demonstrated that 1) the release of purines from the caudal artery was reduced by rubbing the lumen of the artery, and that 2) noradrenaline induced the release of purines in cultured endothelial cells from the caudal artery and thoracic aorta, and the amount of release from the former was larger than that from the latter. Taken together, these findings suggest that the vascular endothelial cells are able to release adenyl purines by an $\alpha_{1}$-adrenoceptor-mediated mechanism and seem not to be homogeneously distributed in the vasculature. As one possibility, the density and the mode of distribution of the $\alpha_{1}$-adrenoceptor on the endothelial cells mediating adenyl purine release may be different in the vasculature.

The adenyl purines released from the primary cultures of endothelial cells from the caudal artery of rats by noradrenaline were mostly nucleotides, mainly ATP. However, in intact caudal artery preparations, the amount of adenosine released was $46 \%$ of the total amount of adenyl purines released. It has been reported that the quantity of adenine nucleotides released 
from cultured endothelial cells was larger than the amount of adenosine released, and it was suggested that the degradation of ATP to adenosine by endothelial cell ectonucleotidase is not very rapid (Pearson and Gordon, 1979 ; Gordon et al., 1986). In contrast, those investigators found that ATP is rapidly degraded to adenosine by cultured smooth muscle cells from the pig aorta (Gordon et al., 1989). We showed that the amount of ATP released by noradrenaline from the intact caudal artery of rats was increased approximately 2 -fold by the 5 -nucleotidase inhibitor, $\alpha, \beta$-methylene ADP (Shinozuka et al., 1994). Thus, most of the ATP released from endothelial cells by noradrenaline may be rapidly hydrolyzed to adenosine by ectonucleotidase on the surface of smooth muscle cells. In primary cultures of rat caudal artery smooth muscle cells, we found no significant release of purines induced by noradrenaline (data not shown). Bodin et al. (1991) have also reported that shear stress did not evoke ATP release in cultured vascular smooth muscle cells. These findings suggest that the vascular smooth muscle cells may not possess a significant ATP releasing function, but may be important in the metabolic conversion of ATP to adenosine. In the present study, we observed that the composition of purines released differed somewhat depending on the region of blood vessel. As one possible explanation for this difference, it is considered that the distribution and/or activity of metabolic converting enzyme, for example ectonucleotidase, is not homogenous in the vasculature.

Taken together, our findings confirm that an $\alpha_{1}$-adrenoceptor-mediated mechanism for ATP release exists on the vascular endothelial cells, and the data strongly suggest that functional heterogeneity involving this $\alpha_{1}$-adrenoceptor-mediated release of ATP exists in the vasculature. A heterogeneous anatomical distribution of $\alpha_{1}$-adrenoceptors on vascular endothelial cells may well explain the differences in the noradrenaline-induced ATP release in the six rat blood vessels. ATP and ADP can produce endothelium-dependent vasodilatation via $\mathrm{P}_{2 \mathrm{y}}$-purinoceptors in the precontracted arteries, and the metabolite of ATP, adenosine, can also produce direct vasodilatation by cAMP accumulation in the arterial smooth muscle cells via $\mathrm{P}_{1}\left(\mathrm{~A}_{2}\right)$-purinoceptor (Dalziel and Westfall, 1994). Both ATP and adenosine reduced noradrenaline release from vascular sympathetic nerves via $\mathrm{P}_{3}$-purinoceptors (Shinozuka et al., 1991). Endogenous adenyl purines released from the arteries may thus produce vasodilatation via $\mathrm{P}_{2 \mathrm{y}^{-}}, \mathrm{P}_{1}\left(\mathrm{~A}_{2}\right)^{-}$, and $\mathrm{P}_{3}$-purinoceptor stimulation, and might control the blood circulation and blood pressure. Indeed, we have shown that ATP and its metabolites released from vascular endothelium are related to the control of blood pressure (Hashimoto et al., 1995). Based on these observations, it is suggested that the regional difference of endothelium-derived ATP release may be associated with the control of the local blood circulation and blood pressure.

\section{Acknowledgements}

This research was supported in part by grants from the Ministry of Education, Science and Culture of Japan; the Smoking Research Foundation of Japan and the National Institutes of Health (HL38126). 


\section{References}

Bodin, P., Bailey, D. and Burnstock, G. (1991). Increased flow-induced ATP release from isolated vascular endothelial cells but not smooth muscle cells. Br. J. Pharmacol. 103 : 1203-1205.

Burnstock, G. (1991). Distribution and roles of purinoceptor subtypes. Nucleosides Nucleotides 10 : 917-930.

Dalziel, H.H. and Westfall, D.P. (1994). Receptors for adenine nucleotides and nucleosides: Subclassification, distribution, and molecular characterization. Pharmacol. Rev. 46: 449466.

Gordon, J.L. (1986). Extracellular ATP: effects, sources and fate. Biochem. J. 233 : 303-319.

Gordon, E.L., Pearson, J.D., and Slakey, L.L. (1986). The hydrolysis of extracellular adenine nucleotides by cultured endothelial cells from pig aorta. J. Biol. Chem. 261 : 15496-15504.

Gordon, E.L., Pearson, J.D., Dickinson, E.S., Moreau, D. and Slakey, L.L. (1989). The hydrolysis of extracellular adenine nucleotides by arterial smooth muscle cells : regulation of adenosine production at the cell surface. J. Biol. Chem., 264: 18986-18992.

Hashimoto, M., Hara, T., Honda, M., Ishinaga, Y., Moriyama, K. and Masumura, S. (1992). Metabolic aspects of endothelial cells cultured from rat aorta. Artery 19: 284-296.

Hashimoto, M., Shinozuka, K., Bjur, R.A., Westfall, D.P., Hattori, K. and Masumura, S. (1995). The effects of age on the release of adenine nucleosides and nucleotides from rat caudal artery. J. Physiol. (Lond.) 489: 841-848.

Mohri, K., Takeuchi, K., Shinozuka, K., Bjur, R.A. and Westfall, D.P. (1993). Simultaneous determination of nerve-induced adenine nucleotides and nucleosides released from rabbit pulmonary artery. Anal. Biochem. 210 : 262-267.

Pearson, J.D. and Gordon, J.L. (1979). Vascular endothelial and smooth muscle cells in culture selectivity release adenine nucleotides. Nature 281 : 384-386.

Sneddon, P. and Westfall D.P. (1984). Pharmacological evidence that adenosine triphosphate and noradrenaline are co-transmitters in the guinea-pig vas deferens. J. Physiol. (Lond.) 347 : 561-580.

Sneddon, P. and Burnstock, G. (1985). ATP as a co-transmitter in rat tail artery. Eur. J. Pharmacol. 106 : 149-152.

Su, C. (1975). Neurogenic release of purine compounds in blood vessels. J. Pharmacol. Exp. Ther. 195 : $159-166$.

Su, C. (1983). Purinergic neurotransmission and neuromodulation. Annu. Rev. Pharmacol. Toxicol. 23 : 397-411.

Shinozuka, K., Sedaa, K.O., Bjur, R.A. and Westfall, D.P. (1991). Participation by purines in the modulation of norepinephrine release by methoxamine. Eur. J. Pharmacol. 192 : 431-434.

Shinozuka, K., Hashimoto, M., Masumura, S., Bjur, R.A., Westfall, D.P. and Hattori, K. (1994). In vitro studies of release of adenine nucleotides and adenosine from rat vascular endothelium in response to $\alpha_{1}$-adrenoceptor stimulation. Br. J. Pharmacol . 113: 1203-1208.

Westfall, D.P., Sedaa, K.O. and Bjur, R.A. (1987). Release of endogenous ATP from rat caudal artery. Blood Vessels 24 : 125-127.

White, T.D. (1988). Role of adenine compounds in autonomic neurotransmission. Pharmacol. Ther. 38 : $129-168$. 